\title{
Immune-to-brain signalling: the role of cerebral CD163-positive macrophages
}

\author{
Ian Galea ${ }^{*}$, Leigh M Felton ${ }^{*}$, Sara Waters ${ }^{*}$, Nico van Rooijen ${ }^{\dagger}, \mathrm{V}$ Hugh Perry ${ }^{*}$, Tracey A \\ Newman $^{*}$
}

* CNS Inflammation Group, School of Biological Sciences, University of Southampton, Bassett Crescent East, Southampton SO16 7PX, UK

${ }^{\dagger}$ Department of Molecular Cell Biology, Vrije Universiteit Medical Centre, Van der Boechorststraat 7, 1081 BT Amsterdam, The Netherlands

Text pages: 6

Figures: 4

\section{Corresponding author:}

Ian Galea

CNS Inflammation Group

School of Biological Sciences

University of Southampton

Bassett Crescent East

Southampton SO16 7PX

UK

Tel 00447870370076

Fax 00442380798793

I.Galea@soton.ac.uk

\section{Acknowledgements:}

We thank the following people for advice, technical assistance and reagents: P. Prescott, N. Platt and M. Cuttle (University of Southampton). This work was supported by EU grant QLG3-CT-2002-00612 (IG), EU COST Action BM0603 (IG, VHP) and MS Society grant 784/03 (LMF). 


\section{Keywords:}

blood brain barrier, macrophages, lipopolysaccharide, cytokines, inflammation 


\begin{abstract}
Systemic inflammation induces cytokine synthesis within the central nervous system. This results in sickness behaviour and may exacerbate ongoing neuroinflammatory disease. The precise mechanisms underlying the relay of signal from the periphery to the central nervous system are not entirely understood. CD163-positive macrophages occupy a unique position at the blood-brain barrier and upregulate prostaglandin-synthesizing enzymes in response to systemic inflammation. This finding suggests that they might play a role in signalling inflammation to the central nervous system. However, here we demonstrate that de novo brain cytokine transcription during systemic endotoxaemia may be prostaglandin-independent. We therefore set out to interrogate more directly the role of CD163-positive macrophages in immune-to-brain signalling. Intracerebroventricular injections of clodronate liposomes were used to selectively deplete CD163-positive macrophages. We show that de novo brain cytokine synthesis during systemic endotoxaemia persists in the absence of CD163-positive macrophages. Cerebral endothelial cells outnumber CD163-positive macrophages and are arguably better situated to signal circulating inflammatory stimuli to the brain.
\end{abstract}


The transfer of an inflammatory message across the blood-brain barrier (BBB) is important in a range of biological responses to ongoing systemic infection or inflammation in both health and disease. It occurs by means of three main mechanisms: (1) direct entry of circulating inflammatory molecules or cells [2,8], (2) signalling via vagal or somatic sensory afferent projections [11], and (3) a cascade of cellular activation starting at the immune-brain interface which progressively penetrates deeper layers of the brain parenchyma. Such a biphasic or multiphasic process has been described at three anatomical areas representing the immune-brain interface: cerebral blood vessels, meninges and circumventricular organs $[3,17,20,27]$, and is evidenced by a delayed and distinct cytokine transcriptional programme [21].

Over the past few years various groups have demonstrated a key role of cerebral endothelial cells in "sensing" the circulating inflammatory milieu, upregulating cyclooxygenase 2 (COX2), and triggering the chain of events resulting in activation of brain parenchyma [5,9,23,26,28,30,34], presumably via prostanglandin E2 (PGE2) synthesis. We wanted to examine the role of CD163-positive macrophages in this process. These cells lie abluminal to the cerebral endothelium, or in the meninges itself, and are constitutively activated [13]. CD163-positive macrophages have been shown to upregulate COX2 during systemic inflammatory challenge $[10,28,30]$. In some studies intravenously administered material was endocytosed by these cells, suggesting that they have access to the circulating milieu [4,22]. In other studies COX2 upregulation occurred in CD163-positive macrophages, but not endothelial cells, after administration of low doses of endotoxin [10,30]. These studies raised the possibility that CD163-positive macrophages might participate in immune-to-brain signalling either by directly sampling 
the circulation, or by acting as amplifiers of the endothelial response. Ultimately cytokines in the brain parenchyma are synthesized by glia and neurons, the major source being microglia [29].

We therefore set out to directly interrogate the role of CD163-positive macrophages by selectively depleting them from a specific region within the forebrain of rats. In all experiments, lipopolysaccharide (LPS E. coli 0111:B4, Sigma, Poole, UK) was administered to model systemic inflammation and de novo cytokine transcription in the right frontal cortex was studied.

Adult male Wistar rats (Harlan, UK) were used in this study under Home Office Licence and in accordance with the Animals (Scientific Procedures) Act 1986, UK. A radio-telemetry system (Data Sciences International, USA) was used to remotely monitor core body temperature of rats two weeks after intraperitoneal implantation of thermistors. ICV injections were performed using a minimally invasive stereotaxic procedure causing mild and transient inflammation [1]. Animal perfusion, tissue processing, semiquantitative real-time polymerase chain reaction (RT-PCR) (Applied Biosystems, UK), enzyme-linked immunosorbent assay (ELISA) (R\&D Systems, UK), immunohistochemistry/flourescence (ED1 or ED2, 1:200, Serotec, UK), microscopy and quantification were performed as described previously [15,16]. Statistical analysis was performed in SPSS v12.

To deplete cerebral CD163-positive macrophages, clodronate (a gift of Roche Diagnostics GmbH, Mannheim, Germany) or phosphate-buffered saline (PBS)-loaded liposomes were prepared as described before [31]. Progressive phagocytosis of liposomes by CD163-positive macrophages leads to a lethal rise in intracellular clodronate 
concentration and consequent apoptosis [32]. 50 $\mu$ l clodronate or PBS-containing liposomes were infused into the 4 th ventricle (coordinates: bregma $-11.6 \mathrm{~mm}$, lateral 0 , depth $8.2 \mathrm{~mm}$ ) over $25 \mathrm{~min}$. This resulted in selective and complete depletion of cerebral CD163-positive macrophages between days 2 and 10 (Fig. 2A-D), as previously reported [25]. We wanted to ensure that this technique did not affect peripheral macrophages. Although there was no reduction in the density of hepatic and splenic red pulp macrophages in the second week after the ICV infusion (Fig. 2E), the morphology of a significant proportion of these cells in the clodronate liposome treated animals led us to suspect that they might not be functionally normal, and we wondered whether the ICVinfused liposomes might be draining to the periphery. A direct communication is known to exist between the subarachnoid space and the peripheral circulation through the cribriform plate, nasal lymphatics, deep cervical lymph nodes and thence into the blood [19,33]. Indeed, ICV infusion of DiI-labelled liposomes revealed their drainage to cervical lymph nodes (Fig. 2F), spleen (Fig. 2G) and liver (Fig. 2H) and double immunofluorescence showed uptake of liposomes by macrophages in these organs (Fig. 2I,J). We therefore used ELISA to measure the serum cytokine response after systemic endotoxin challenge ( $200 \mu \mathrm{g} / \mathrm{kg}$ lipopolysaccharide intraperitoneally) on day 9 after ICV liposome infusion. We found that serum levels of TNF $\alpha$, IL1 $\beta$ and IL6 were significantly lower in clodronate versus control liposome treated animals and a dose-response effect was seen (Fig. 3A). Lower doses of clodronate liposomes did not affect the serum cytokine response to systemic endotoxin challenge but this was accompanied by unacceptably low CD163-positive macrophage depletion (Fig. 3B). Several attempts at technique modification were therefore undertaken. It was found that infusion of $15 \mu 1$ 
concentrated suspension of clodronate-loaded liposomes, diluted to $25 \mu$, in the right lateral ventricle (coordinates: bregma $-0.8 \mathrm{~mm}$, lateral $1.4 \mathrm{~mm}$, depth $4.4 \mathrm{~mm}$ ) of rats over 12.5 minutes did not affect the serum cytokine response to systemic endotoxin challenge on day 9 after the infusion, as compared to control liposomes (Fig. 3C). This optimized technique resulted in a 65\% depletion of CD163-positive macrophages in the septum, $1.8 \mathrm{~mm}$ rostral to the infusion site, but a more focal almost complete depletion in the right frontoparietal cortex and striatum (98\% and 95\% respectively, $\mathrm{n}=5$ for both control and clodronate liposome groups). All meningeal CD163-positive macrophages throughout the brain were depleted. This protocol was used in all subsequent experiments, during which we limited analysis of brain cytokine induction to the right frontoparietal cortex, which also had the advantage of avoiding brain regions which are known to be activated directly or indirectly by vagal projections from the periphery during systemic inflammatory challenge [11].

In order to study immune-to-brain signalling, we administered endotoxin to induce an episode of systemic inflammation. Intravenous injection of $500 \mu \mathrm{g} / \mathrm{kg}$ LPS was used in order to achieve less variable and clear-cut central cytokine and fever responses. Rat cage activity decreased and animals exhibited a hunched posture, piloerection and rigors. Hyperthermia was seen in saline- pretreated animals $2 \frac{1}{2}$ hours after endotoxin challenge. Indomethacin pre-treatment $(15 \mathrm{mg} / \mathrm{kg}$ i.p.) 30 minutes prior to systemic endotoxin challenge resulted in complete suppression of this fever when compared to vehicle (Fig. 1A). Indomethacin administration also resulted in a mild hypothermia; this phenomenon has been previously reported [14]. 
Systemic inflammation is accompanied by a delayed de novo expression of cytokines behind the BBB [21]. Indeed when intact animals were challenged systemically with endotoxin, we observed significant transcription of TNF $\alpha$, IL1 $\beta$ and IL6 mRNA in the frontal cortex using RT-PCR; none occurred in animals receiving vehicle (Fig. 1B). To determine whether this was $\mathrm{PGE}_{2}$-dependent we examined the cerebral cytokine response in animals pre-treated with indomethacin $(15 \mathrm{mg} / \mathrm{kg})$ or vehicle. This represents a high dose of indomethacin and is sufficient to result in complete suppression of fever (see above) and inducible $\mathrm{PGE}_{2}$ synthesis [12]. Surprisingly, we observed that indomethacin did not affect the de novo transcription of IL1 $\beta$ seen in the brain after systemic endotoxin challenge in this model (Fig. 1C); TNF $\alpha$ and IL6 transcription was not suppressed though a non-significant decrease was observed. These results indicated that a significant amount of cytokine induction behind the BBB in response to systemic inflammation was prostaglandin-independent.

The prevention of hyperthermia as a consequence of pre-treatment with indomethacin prior to the endotoxin challenge suggested that a sufficient amount of the drug was absorbed and available to the immune-brain interface. However de novo brain cytokine induction still occurred after systemic endotoxin challenge in the indomethacin treated animals. Yet, a functional role for cells at the BBB has been inferred on the basis of COX2 and microsomal $\mathrm{PGE}_{2}$-synthase expression [5,9,23,26,28,30,34]. We therefore set out to use a different and more direct method to investigate the role of CD163positive macrophages in immune-to-brain signalling by selectively depleting this population and studying the effect of this depletion on the induction of cerebral cytokine synthesis in response to peripheral inflammation. 
It was first established that ICV infusion of control liposomes per se did not affect the central cytokine response to systemic endotoxin challenge (Fig. 4A). Then, animals received a clodronate or control liposome ICV infusion, and 9 days later were challenged with endotoxin systemically. Both groups of animals exhibited similar clinical symptoms of endotoxaemia, as described above. No significant differences in TNF $\alpha$, IL1 $\beta$ and IL6 mRNA levels in the frontoparietal cortex were detected between clodronate and control liposome treated animals (Fig. 4B). This shows that cerebral CD163-positive macrophages do not have an essential role in the induction of cytokine synthesis within the CNS during systemic inflammation. Cytokine mRNA upregulation may occur in the absence of protein synthesis. We therefore measured IL $1 \beta$ protein by ELISA in the right frontoparietal cortex of endotoxin-challenged animals 9 days after ICV clodronate or control liposome infusion. This confirmed that message was being translated into protein; no significant difference in IL1 $\beta$ protein was observed between clodronate and control liposome treated animals (Fig. 4C).

The aim of the current study was to determine whether CD163-positive macrophages at the BBB have an essential role in the relay process of an inflammatory stimulus between the periphery and the CNS. In order to do this we carried out a functional study in which we determined the impact of depleting CD163-positive macrophages on cytokine induction within the brain during systemic inflammation. Regionally specific depletion was achieved by using an ICV infusion of clodronateloaded liposomes. We show that the brain cytokine response to systemic inflammation occurred despite depletion of CD163-positive macrophages. We conclude that CD163- 
positive macrophages are not essential for immune-to-brain signalling in the healthy brain.

Excluding a role, as compared to proving it, is always difficult. It is of course still possible that a major signalling role of CD163-positive macrophages was taken over by endothelial cells after depletion, or that the very small population of CD163-positive perivascular macrophages (2\%) still present after depletion might have been enough to play a signalling role. Circumstantial evidence suggests that this is unlikely. For instance, the early CNS inflammatory response was significantly reduced in bone marrow chimeras three months after transfer of wild-type bone marrow into toll-like receptor 4 knockout mice, at which stage such mice would be expected to have LPS-responsive cerebral CD163-positive macrophages but LPS-resistant endothelial cells [6]. In addition we estimate that the density of CD163-positive macrophages in rat brain is 25 fold lower than that of endothelial cells (data not shown), which form a continuous sheet of cells directly in contact with the circulation, excluding CD163-positive macrophages from the blood-brain interface.

Both endothelial cells and CD163 macrophages show upregulation of COX2 expression in response to a systemic immune challenge, thus implicating them in communication of the inflammatory message to the brain. However here we demonstrate that COX2 upregulation is not essential in the relay of the message as indomethacin pretreatment, sufficient to inhibit fever induction, did not abolish de novo cytokine expression in the brain during systemic inflammation. Prostaglandins have been shown to mediate several components of sickness behaviour in response to systemic inflammation: fever [11], hyperalgesia [18], activation of the HPA axis [24] and social interaction [7]. 
All these responses can be inhibited by NSAIDs. Here we unearth a clear disparity by showing that de novo cytokine expression within the brain after systemic endotoxin challenge is prostaglandin-independent. The experiments presented here therefore examine a prostaglandin-independent immune-to-brain pathway distinct from that subserved by the circumventricular organs and hypothalamus, which constitute an important immune-brain interface driving the prostaglandin-dependent responses mentioned above.

Finally, this study examines immune-brain signalling in the healthy brain, but CD163-positive macrophages accumulate in response to pathology within the brain [16] and their role in immune-to-brain signalling in the diseased brain remains to be determined. 


\section{Figure legends}

Fig. 1. Fever generation after systemic endotoxin challenge is prostaglandin-dependent but not de novo brain cytokine transcription is prostaglandin-independent. $\boldsymbol{A}$, Core body temperature was measured telemetrically in two groups of rats ( $\mathrm{n}=5$ each) which were pre-treated with an i.p. dose of indomethacin $(15 \mathrm{mg} / \mathrm{kg})$ or vehicle respectively, 30 minutes before receiving $500 \mu \mathrm{g} / \mathrm{kg}$ LPS i.v. Indomethacin abolished the hyperthermia seen in rats pre-treated with vehicle, and lowered temperature below baseline (preceding day). $\boldsymbol{B}$, Rats were challenged with $500 \mu \mathrm{g} / \mathrm{kg}$ LPS or saline i.v. ( $\mathrm{n}=5$ per group) and right frontoparietal cortex was harvested 3 hours later for cytokine mRNA analysis by RTPCR. An upregulation of TNF $\alpha(p<0.001), \operatorname{IL} 1 \beta(p=0.008)$ and IL6 $(p=0.003)$ was seen (Student's t-test). $\boldsymbol{C}$, Two groups of rats ( $\mathrm{n}=6$ each) were pre-treated with an i.p. dose of indomethacin $(15 \mathrm{mg} / \mathrm{kg})$ or vehicle respectively, 30 minutes before receiving $500 \mu \mathrm{g} / \mathrm{kg}$ LPS i.v. Cortical tissue samples were harvested 3 hours later and analyzed by RT-PCR for TNF $\alpha$, IL1 $\beta$, and IL6 mRNA. No significant differences in cytokine mRNA levels were seen ( $\mathrm{p}>0.05$ by Mann-Whitney test).
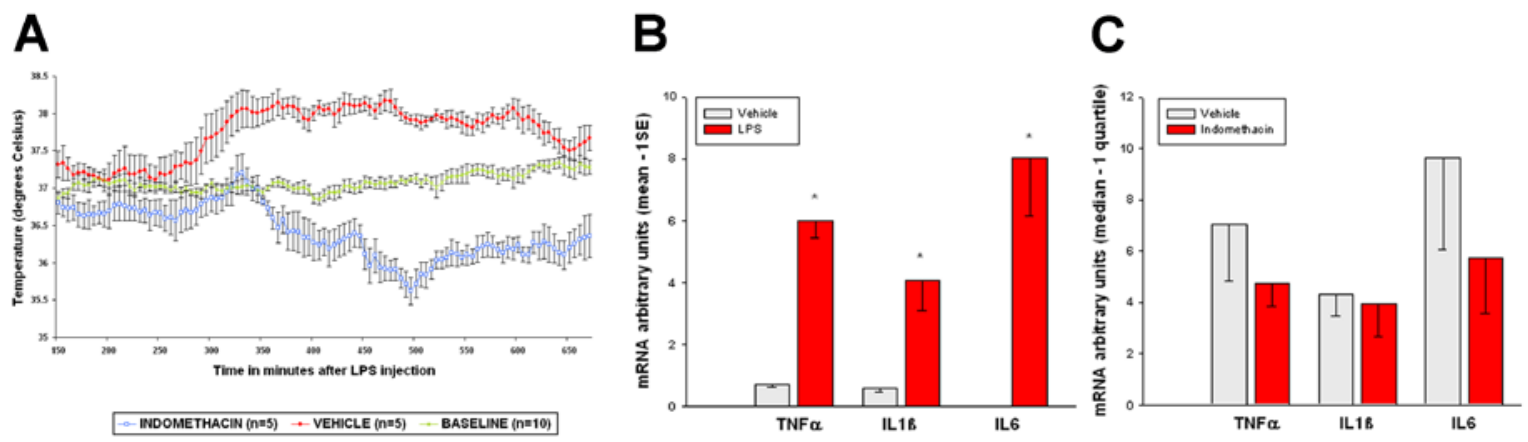

Figure 1 
Fig. 2. An intracerebroventricular liposome technique to deplete cerebral CD163-positive macrophages. A-D, Rats received ICV infusions of 50 $\mu$ l control (A-B) or clodronate liposomes ( $\boldsymbol{C}-\boldsymbol{D})$ (n=3 per group) and were perfused 9 days later. Brain sections were processed for ED2 immunohistochemistry using DAB (brown) as chromogen. Complete perivascular $(\boldsymbol{A}, \boldsymbol{C})$ and meningeal $(\boldsymbol{B}, \boldsymbol{D})$ CD163-positive macrophage depletion was seen in clodronate-treated animals $(\boldsymbol{C}, \boldsymbol{D})$ but not in sham-treated controls $(\boldsymbol{A}, \boldsymbol{B})$. $\boldsymbol{E}$, ED2positive Kupffer cells in liver ( $n=3$ per group) and red pulp macrophages in spleen ( $n=5$ per group) were quantified on day 9 after ICV infusion of $50 \mu$ l clodronate or control liposomes: no statistically significant difference was seen (Student's t-test: $\mathrm{p}=0.384$ for liver and p=0.248 for spleen). $\boldsymbol{F}$-J, Two groups of 3 rats received an ICV infusion of 50 $\mu 1$ DiI-labelled clodronate liposomes and were perfused after 3 and 9 days respectively. Tissue sections were processed for immunofluorescence. Representative cervical lymph node $(\boldsymbol{F})$, spleen $(\boldsymbol{G})$ and liver $(\boldsymbol{H})$ sections from animals perfused at 9 days show drainage to these organs (red: DiI, blue: DAPI nuclear counterstain). Double immunofluorescence followed by confocal microscopy in $0.43 \mu \mathrm{m}$ optical slices revealed the liposomes to be within macrophages (I, green ED2 as macrophage surface marker), specifically in the lysosomal compartment ( $\boldsymbol{J}$, green ED1 as lysosomal marker). Scale bar: $\boldsymbol{A}-\boldsymbol{D}, 20 \mu \mathrm{m} ; \boldsymbol{F}, 200 \mu \mathrm{m} ; \boldsymbol{G}, 40 \mu \mathrm{m} ; \boldsymbol{H}, 100 \mu \mathrm{m} ; \mathbf{I}-\boldsymbol{J}, 5 \mu \mathrm{m}$. 

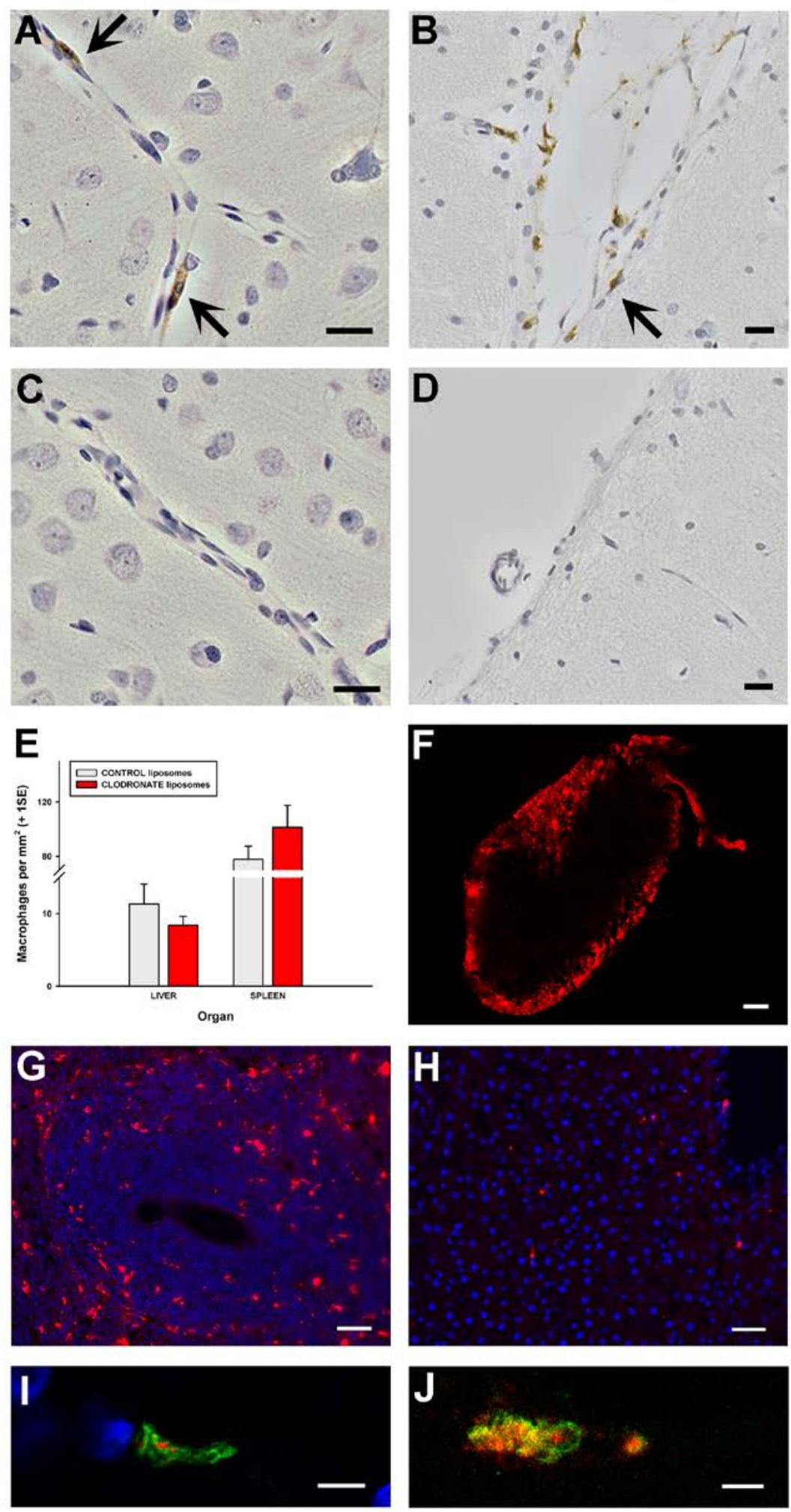

Figure 2 
Fig. 3. Optimization of an intracerebroventricular liposome technique to deplete cerebral CD163-positive macrophages. $\boldsymbol{A}, \boldsymbol{B}$, Rats received ICV infusions of several doses of clodronate ( $\mathrm{n}=13$ total) or control liposomes ( $\mathrm{n}=12$ total) followed by an i.p. injection of $200 \mu \mathrm{g} / \mathrm{kg}$ LPS. Brains and serum were collected 21/2 hours later. Brain sections at bregma $2 \mathrm{~mm}$ were processed for ED2 immunohistochemistry of the septum. Serum TNF $\alpha$ was assayed by ELISA. A dose-response was seen for both serum TNF $\alpha(\boldsymbol{A})$ and CD163positive macrophages in the septum $(\boldsymbol{B})$ (one-way ANOVA: $\mathrm{p}<0.001$; Bonferroni post hoc comparison: $\mathrm{p}<0.05$ for $20 \mu 1\left[^{*}\right]$ and $\mathrm{p}<0.01$ for $\left.50 \mu 1\left[^{* *}\right]\right)$. $C$, Serum TNF $\alpha$ levels $2 \frac{1}{2}$ hours after $200 \mu \mathrm{g} / \mathrm{kg}$ LPS i.p. in animals treated with clodronate or control liposomes ( $\mathrm{n}=5$ per group) using the optimized method (see text) 9 days previously. No statistically significant difference was seen (Student's t-test: $\mathrm{p}=0.582$ ).
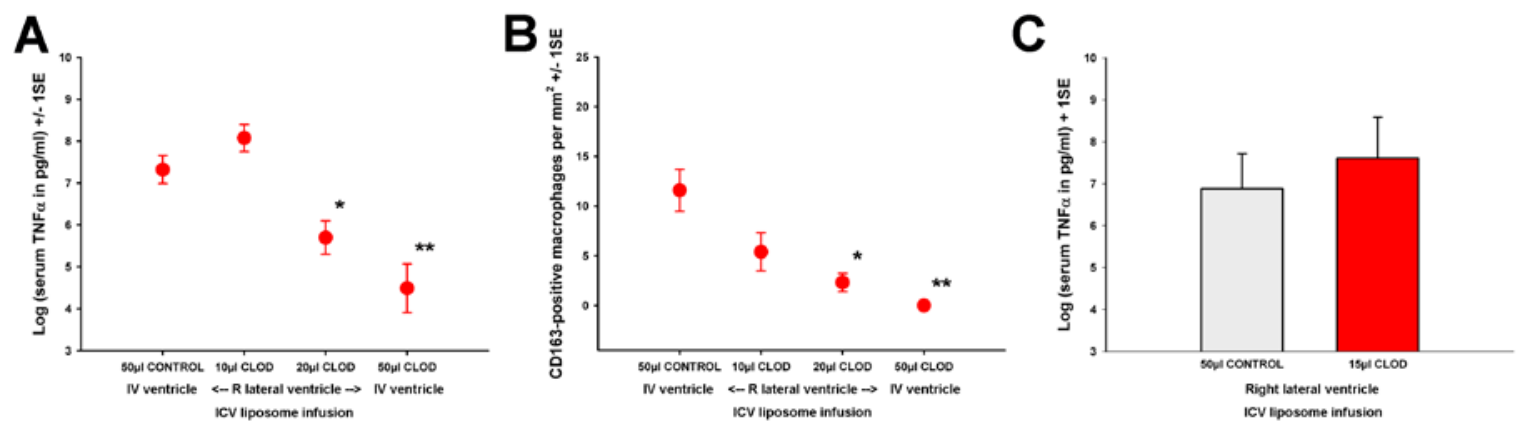

Figure 3

Fig. 4. Brain cytokine induction after systemic endotoxin challenge: the role of cerebral CD163-positive perivascular macrophages. $\boldsymbol{A}$, Cytokine mRNA upregulation in right frontoparietal cortex after an i.v. challenge of $500 \mu \mathrm{g} / \mathrm{kg}$ LPS is not affected by ICV infusion of control liposomes 9 days previously compared to naïve rats ( $n=5$ per group). No statistically significant difference was observed in TNF $\alpha(p=0.751), \operatorname{IL} 1 \beta(p=0.895)$ 
and IL6 ( $\mathrm{p}=0.918$ ) mRNA between groups (Student's t-test). $\boldsymbol{B}$, Right frontoparietal cortex cytokine mRNA levels (by RT-PCR) 3 hours after $500 \mu \mathrm{g} / \mathrm{kg}$ LPS i.v. in animals treated with clodronate or control liposomes ( $\mathrm{n}=4$ per group) using the optimized method (see text) 9 days previously. No statistically significant difference was observed in TNF $\alpha$ $(\mathrm{p}=0.782), \operatorname{IL} 1 \beta(\mathrm{p}=0.084)$ and IL6 $(\mathrm{p}=0.920)$ mRNA between groups (Student's t-test). $C$, Right frontoparietal cortex IL1 $\beta$ protein levels (by ELISA) 3 hours after $500 \mu \mathrm{g} / \mathrm{kg}$ LPS i.v. in animals receiving ICV infusion of clodronate $(\mathrm{n}=6)$ or control liposomes $(\mathrm{n}=7)$ in the right lateral ventricle using the optimized method (see text) 9 days previously. No statistically significant difference was seen (Student's t-test: $\mathrm{p}=0.564)$. No IL1 $\beta$ was detected after i.v. saline injection of rats receiving control liposomes ICV 9 days previously.
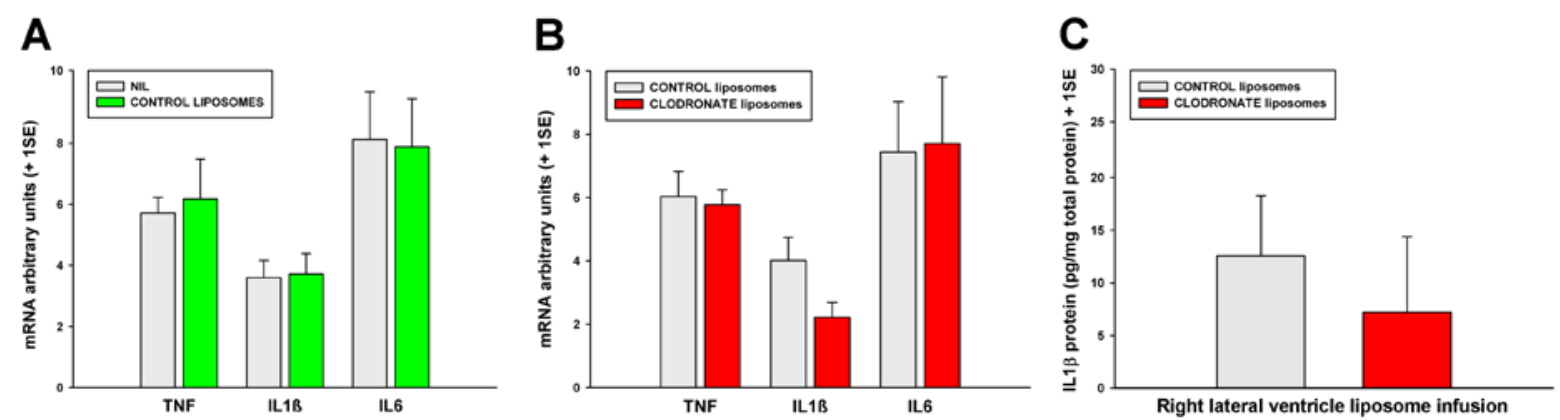

Figure 4 


\section{Reference List}

[1] P.B. Andersson, V.H. Perry, S. Gordon, The acute inflammatory response to lipopolysaccharide in CNS parenchyma differs from that in other body tissues, Neuroscience 48 (1992) 169-186.

[2] W.A. Banks, A.J. Kastin, R.D. Broadwell, Passage of cytokines across the bloodbrain barrier, Neuroimmunomodulation. 2 (1995) 241-248.

[3] C.D. Breder, C. Hazuka, T. Ghayur, C. Klug, M. Huginin, K. Yasuda, M. Teng, C.B. Saper, Regional induction of tumor necrosis factor alpha expression in the mouse brain after systemic lipopolysaccharide administration, Proc Natl Acad Sci U S A 91 (1994) 11393-11397.

[4] R.D. Broadwell, M. Salcman, Expanding the definition of the blood-brain barrier to protein, Proc. Natl. Acad. Sci. U. S. A 78 (1981) 7820-7824.

[5] C. Cao, K. Matsumura, K. Yamagata, Y. Watanabe, Endothelial cells of the rat brain vasculature express cyclooxygenase-2 mRNA in response to systemic interleukin-1 beta: a possible site of prostaglandin synthesis responsible for fever, Brain Res 733 (1996) 263-272.

[6] S. Chakravarty, M. Herkenham, Toll-like receptor 4 on nonhematopoietic cells sustains CNS inflammation during endotoxemia, independent of systemic cytokines, J Neurosci. 25 (2005) 1788-1796. 
[7] F. Crestani, F. Seguy, R. Dantzer, Behavioural effects of peripherally injected interleukin-1: role of prostaglandins, Brain Res. 542 (1991) 330-335.

[8] J. Davidson, H.T. Abul, A.S. Milton, D. Rotondo, Cytokines and cytokine inducers stimulate prostaglandin E2 entry into the brain, Pflugers Arch 442 (2001) 526-533.

[9] M. Ek, D. Engblom, S. Saha, A. Blomqvist, P.J. Jakobsson, D. Ericsson, Inflammatory response: pathway across the blood-brain barrier, Nature 410 (2001) 430-431.

[10] J.K. Elmquist, C.D. Breder, J.E. Sherin, T.E. Scammell, W.F. Hickey, D. Dewitt, C.B. Saper, Intravenous lipopolysaccharide induces cyclooxygenase 2-like immunoreactivity in rat brain perivascular microglia and meningeal macrophages, J Comp Neurol 381 (1997) 119-129.

[11] J.K. Elmquist, T.E. Scammell, C.B. Saper, Mechanisms of CNS response to systemic immune challenge: the febrile response, Trends Neurosci. 20 (1997) 565-570.

[12] A.S. Fabricio, F.H. Veiga, R. Cristofoletti, P. Navarra, G.E. Souza, The effects of selective and nonselective cyclooxygenase inhibitors on endothelin-1-induced fever in rats, Am. J. Physiol Regul. Integr. Comp Physiol 288 (2005) R671-R677.

[13] B.O. Fabriek, I. Galea, V.H. Perry, C.D. Dijkstra, Cerebral perivascular macrophages and the blood brain barrier. In: H.E. de Vries, A. Prat (Eds.), The 
blood-brain barrier and its microenvironment: basic physiology to neurological disease, Taylor and Francis, New York, 2005, pp. 295-316.

[14] W. Feldberg, P.N. Saxena, Prostaglandins, endotoxin and lipid A on body temperature in rats, J Physiol 249 (1975) 601-615.

[15] L.M. Felton, C. Cunningham, E.L. Rankine, S. Waters, D. Boche, V.H. Perry, MCP-1 and murine prion disease: separation of early behavioural dysfunction from overt clinical disease, Neurobiol. Dis. 20 (2005) 283-295.

[16] I. Galea, K. Palin, T.A. Newman, N. van Rooijen, V.H. Perry, D. Boche, Mannose receptor expression specifically reveals perivascular macrophages in normal, injured, and diseased mouse brain, Glia 49 (2005) 375-384.

[17] M. Herkenham, H.Y. Lee, R.A. Baker, Temporal and spatial patterns of c-fos mRNA induced by intravenous interleukin-1: a cascade of non-neuronal cellular activation at the blood-brain barrier, J Comp Neurol 400 (1998) 175-196.

[18] T. Hori, T. Oka, M. Hosoi, M. Abe, K. Oka, Hypothalamic mechanisms of pain modulatory actions of cytokines and prostaglandin E2, Ann. N. Y. Acad. Sci. 917 (2000) 106-120.

[19] S. Kida, R.O. Weller, E.T. Zhang, M.J. Phillips, F. Iannotti, Anatomical pathways for lymphatic drainage of the brain and their pathological significance, Neuropathol Appl Neurobiol 21 (1995) 181-184. 
[20] S. Lacroix, D. Feinstein, S. Rivest, The bacterial endotoxin lipopolysaccharide has the ability to target the brain in upregulating its membrane CD14 receptor within specific cellular populations, Brain Pathol. 8 (1998) 625-640.

[21] S. Laye, P. Parnet, E. Goujon, R. Dantzer, Peripheral administration of lipopolysaccharide induces the expression of cytokine transcripts in the brain and pituitary of mice, Brain Res Mol Brain Res 27 (1994) 157-162.

[22] M. Mato, S. Ookawara, A. Sakamoto, E. Aikawa, T. Ogawa, U. Mitsuhashi, T. Masuzawa, H. Suzuki, M. Honda, Y. Yazaki, E. Watanabe, J. Luoma, S. YlaHerttuala, I. Fraser, S. Gordon, T. Kodama, Involvement of specific macrophagelineage cells surrounding arterioles in barrier and scavenger function in brain cortex, Proc. Natl. Acad. Sci. U. S. A 93 (1996) 3269-3274.

[23] K. Matsumura, C. Cao, M. Ozaki, H. Morii, K. Nakadate, Y. Watanabe, Brain endothelial cells express cyclooxygenase-2 during lipopolysaccharide-induced fever: light and electron microscopic immunocytochemical studies, J Neurosci 18 (1998) 6279-6289.

[24] A. Morimoto, N. Murakami, T. Nakamori, Y. Sakata, T. Watanabe, Possible involvement of prostaglandin E in development of ACTH response in rats induced by human recombinant interleukin-1, J Physiol 411 (1989) 245-256.

[25] M.M. Polfliet, P.H. Goede, E.M. Kesteren-Hendrikx, N. van Rooijen, C.D. Dijkstra, T.K. van den Berg, A method for the selective depletion of perivascular 
and meningeal macrophages in the central nervous system, J. Neuroimmunol. 116 (2001) 188-195.

[26] N. Quan, M. Whiteside, M. Herkenham, Cyclooxygenase 2 mRNA expression in rat brain after peripheral injection of lipopolysaccharide, Brain Res 802 (1998) 189-197.

[27] N. Quan, M. Whiteside, L. Kim, M. Herkenham, Induction of inhibitory factor kappaBalpha mRNA in the central nervous system after peripheral lipopolysaccharide administration: an in situ hybridization histochemistry study in the rat, Proc. Natl. Acad. Sci. U. S. A 94 (1997) 10985-10990.

[28] S. Rivest, What is the cellular source of prostaglandins in the brain in response to systemic inflammation? Facts and controversies, Mol. Psychiatry 4 (1999) 500507.

[29] N.J. Rothwell, G. Luheshi, S. Toulmond, Cytokines and their receptors in the central nervous system: physiology, pharmacology, and pathology, Pharmacol Ther 69 (1996) 85-95.

[30] J.C. Schiltz, P.E. Sawchenko, Distinct brain vascular cell types manifest inducible cyclooxygenase expression as a function of the strength and nature of immune insults, J Neurosci 22 (2002) 5606-5618.

[31] N. van Rooijen, A. Sanders, Liposome mediated depletion of macrophages: mechanism of action, preparation of liposomes and applications, J. Immunol. Methods 174 (1994) 83-93. 
[32] N. van Rooijen, A. Sanders, T.K. van den Berg, Apoptosis of macrophages induced by liposome-mediated intracellular delivery of clodronate and propamidine, J. Immunol. Methods 193 (1996) 93-99.

[33] R.O. Weller, S. Kida, E.T. Zhang, Pathways of fluid drainage from the brain-morphological aspects and immunological significance in rat and man, Brain Pathol 2 (1992) 277-284.

[34] K. Yamagata, K. Matsumura, W. Inoue, T. Shiraki, K. Suzuki, S. Yasuda, H. Sugiura, C. Cao, Y. Watanabe, S. Kobayashi, Coexpression of microsomal-type prostaglandin E synthase with cyclooxygenase-2 in brain endothelial cells of rats during endotoxin-induced fever, J Neurosci. 21 (2001) 2669-2677. 